\title{
INFLUENCE OF WALL WAVINESS ON FRICTION AND PRESSURE DROP IN CHANNELS
}

\author{
K. VAJRAVELU \\ Department of Mathematics \\ East Carolina University \\ Greenville, North Carolina 27834 \\ and \\ ALI H. NAYFEH \\ Department of Engineering Science and Mechanics \\ Virginia Polytechnic Institute and State University \\ Blacksburg, Virginia 24061 \\ (Received November 7, 1980)
}

ABSTRACT. An attention has been given to investigate the flow behavior of an incompressible viscous fluid confined in horizontal wavy channels and set in motion due to the movement of the upper wall and the pressure differences. The governing equations have been solved analytically as well as numerically subject to the relevant boundary conditions by assuming that the solution consists of two parts: a mean part and a disturbance or perturbed part. For small and moderate Reynolds numbers, the analytical solution for the perturbed part has been found to be in good agreement with the numerical one. The effects of Reynolds number, the pressure gradient parameter, and the undulation wavenumber on friction and pressure drop are found to be quite significant. In addition to the flow behavior for both long and short waves and for large Reynolds numbers, the effect of the wall waviness on friction and pressure drop has been examined for any arbitrary amplitude of the wavy wall.

KEY WORDS AND PHRASES. Incompressible viscous flow, laminar flow, pressure drop, skin friction, and wall waviness.

1980 MATHEMATICS SUBJECT CLASSIFICATION CODES. 760. 


\section{INTRODUCTION.}

There are many physical applications of coupled pressure and shear flows. These include, hydrodynamic lubrication of a sleeve bearing, tube flight vehicles for the development of transportation systems. In addition to having many applications, these flows are of sufficient interest in its own right. They pose no theoretical problems when the fluid motion is laminar, fully developed and confined between parallel flat walls. However, if the shear flows occur between wavy walls, there are certain mathematical difficulties involved with the study of such flows. Consequently, an approximate theoretical treatment may be provided to gain some understanding of shear flow problems.

Literature is replete with theoretical as well as experimental problems dealing with the flow pattern of a viscous fluid confined between flat solid boundaries. Mention may be made of the studies (of these problems) by Berman [1], Balaram [2], Hahn and Kettleborough [3], and Szeri et al. [4]. However, there are many physical situations in which the surfaces of the solid boundaries are wavy in nature. For example, even the surface formed by cleavage of mica contains irregularities of the order of $20 \dot{\mathrm{A}}$ in size, and the irregularities of the surface of an ideally smooth quartz crystal can be up to $100 \dot{\mathrm{A}}$ in height, which have been discussed in detail by Kragelskif [5] .

Although several authors have studied the problems of viscous fluid flows confined between flat boundaries, an attention has been hardly given to these flows in horizontal wavy channels with a moving wall. It seems to us that the findings of the investigations of the fluid flows confined between wavy boundaries will have a definite bearing on the hydrodynamic theory of lubrication, in particular on the bearing industry. So the main objective of this paper is to investigate the effects of wall waviness on friction and pressure drop of the generalized Couette flow. Since the theoretical treatment of the problem is complicated, it is solved by a numerical perturbation technique. The solution consists of two parts: a mean part, corresponding to the fully developed mean flow and a small disturbance. The former coincides with the well known Couette 
flow after modifications resulting from the different non-dimensionalizations employed in classical Couette flow and the present problem. The latter is obtained by the use of the method of superposition coupled with an orthonormalization procedure and a variable-step Runge-Kutta-Fehlberg integration scheme of Scott and Watts [6]. A comparison is made between the numerical and the analytical solutions.

2. FORMULATION AND SOLUTION OF THE PROBLEM.

We consider a channel with the wavy walls represented by $\mathrm{y}^{*}=\mathrm{d}^{*}+\varepsilon^{*} \cos \mathrm{k}^{*} \mathrm{x}^{*}\left(\mathrm{Y}_{1}\right.$ say $)$ and $\mathrm{y}^{*}=-\mathrm{d}^{*}+\mathrm{a}^{*} \cos \left(\mathrm{k}^{*} \mathrm{x}^{*}+\theta\right)\left(\mathrm{Y}_{2}\right.$ say $)$. The representation of the second wall alows us to study the flow behavior in the following four different types of channels by giving the values $0, \pi / 2, \pi$ and $3 \pi / 2$ to $\theta:$ (i) the crest of a wall corresponds to the crest of the other wall of the channel; ( $i i)$ one of the walls considered in ( $i$ ) has a phase-advance/lag; (iii) the crest of a wall corresponds to the trough of the other; and (iv) one of the walls considered in (iii) has a phase-advance/lag.

We make the following assumptions: (a) all fluid properties are constant; (b) the flow is laminar, steady and two-dimensional; and (c) the effects of elastic distortion are neglected (as in reference [3]). Under these assumptions, the equations which govern the steady, two-dimensional motion of a viscous incompressible fluid in the channel are

$$
\begin{aligned}
& \rho *\left(u^{*} \frac{\partial u^{*}}{\partial x^{*}}+v^{*} \frac{\partial u^{*}}{\partial y^{*}}\right)=-\frac{\partial p^{*}}{\partial x^{*}}+\mu *\left(\frac{\partial^{2} u^{*}}{\partial x^{*}}+\frac{\partial^{2} u^{*}}{\partial y^{*} 2}\right), \\
& \rho *\left(u^{*} \frac{\partial v^{*}}{\partial x^{*}}+v^{*} \frac{\partial v^{*}}{\partial y^{*}}\right)=-\frac{\partial p^{*}}{\partial y^{*}}+\mu *\left(\frac{\partial^{2} v^{*}}{\partial x^{*} 2}+\frac{\partial^{2} v^{*}}{\partial y^{*} 2}\right), \\
& \frac{\partial u^{*}}{\partial x^{*}}+\frac{\partial v^{*}}{\partial y^{*}}=0
\end{aligned}
$$

where $\mathrm{u}^{*}$ and $\mathrm{v}^{*}$ are the velocity components, $\mathrm{p}^{*}$ is the pressure, and the other symbols have their usual meanings. The boundary conditions relevant to the problem are taken as

$$
\left.\begin{array}{l}
\mathrm{u}^{*}=\mathrm{U}, \mathrm{v}^{*}=0 \text { on } \mathrm{y}^{*}=\mathrm{d}^{*}+\varepsilon^{*} \cos \mathrm{k}^{*} \mathrm{x}^{*}, \\
\mathrm{u}^{*}=0, \mathrm{v}^{*}=0 \text { on } \mathrm{y}^{*}=-\mathrm{d}^{*}+\alpha^{*} \cos \left(\mathrm{k}^{*} \mathrm{x}^{*}+\theta\right) \cdot \theta
\end{array}\right\}
$$


We define dimensionless variables as

$$
\mathrm{x}=\mathrm{x}^{*} / \mathrm{d}^{*}, \mathrm{y}=\mathrm{y} * / \mathrm{d} *, \mathrm{u}=\mathrm{u} * / \mathrm{U}, \mathrm{v}=\mathrm{v}^{*} / \mathrm{U} \text { and } \mathrm{p}=\mathrm{p} \text { */ } / \mathrm{U}^{2} \text {. }
$$

Then, we rewrite $(2.1)-(2.3)$ and the boundary conditions (2.4) as

$$
\left.\begin{array}{l}
R\left(u \frac{\partial u}{\partial x}+v \frac{\partial u}{\partial y}+\frac{\partial p}{\partial x}\right)=\left(\frac{\partial^{2} u}{\partial x^{2}}+\frac{\partial^{2} u}{\partial y^{2}}\right), \\
R\left(u \frac{\partial v}{\partial x}+v \frac{\partial v}{\partial y}+\frac{\partial p}{\partial y}\right)=\left(\frac{\partial^{2} v}{\partial x^{2}}+\frac{\partial^{2} v}{\partial y^{2}}\right), \\
\frac{\partial u}{\partial x}+\frac{\partial v}{\partial y}=0, \\
u=1, v=0 \text { on } y=1+\varepsilon \cos k x, \\
u=0, v=0 \text { on } y=-1+\alpha \cos (k x+\theta)
\end{array}\right\}
$$

where $R=U d * / \nu^{*}$, the Reynolds number, $\varepsilon=\varepsilon^{*} / \mathrm{d}^{*}$, and $\alpha=\alpha * / \mathrm{d}^{*}$, the dimensionless amplitudes, and $\mathrm{k}=\mathrm{k} * \mathrm{~d} *$, the dimensionless wavenumber.

By the method of perturbations, given by Nayfeh [7], we take the flow field in the form

$$
\left.\begin{array}{lr}
u(x, y)= & u_{0}(y)+u_{1}(x, y), \\
v(x, y)= & v_{1}(x, y), \\
p(x, y)= & p_{0}(x)+p_{1}(x, y)
\end{array}\right\}
$$

where the perturbations $u_{1}, v_{1}$, and $p_{1}$ are small compared with the mean or the zeroth-order quantities. With the help of $(2.9)$, equations $(2.5)-(2.7)$ become

$$
\mathrm{d}^{2} \mathrm{u}_{0} / \mathrm{dy} \mathrm{y}^{2}=\mathrm{RC}
$$

to zeroth-order, and

$$
\begin{aligned}
& R\left(u_{0} \frac{\partial u_{1}}{\partial x}+v_{1} \frac{\partial u_{0}}{\partial y}+\frac{\partial p_{1}}{\partial x}\right)=\left(\frac{\partial^{2} u_{1}}{\partial x^{2}}+\frac{\partial^{2} u_{1}}{\partial y^{2}}\right), \\
& R\left(u_{0} \frac{\partial u_{1}}{\partial x}+\frac{\partial p_{1}}{\partial y}\right)=\left(\frac{\partial^{2} v_{1}}{\partial x^{2}}+\frac{\partial^{2} v_{1}}{\partial y^{2}}\right), \\
& \frac{\partial u_{1}}{\partial x}+\frac{\partial v_{1}}{\partial y}=0
\end{aligned}
$$

to first-order, where $\frac{\partial \mathrm{p}_{0}}{\partial \mathrm{x}}=\mathrm{C}$. Also, with the help of (2.9), the boundary conditions (2.8) can be easily simplified to

$$
\left.\begin{array}{lll}
\mathrm{u}_{0}=1 & \text { on } & \mathrm{y}=1 \\
\mathrm{u}_{0}=0 & \text { on } & \mathrm{y}=-1
\end{array}\right\}
$$




$$
\left.\begin{array}{l}
\mathrm{u}_{1}=-\mathrm{u}_{0}^{\prime}, \mathrm{v}_{1}=0 \text { on } \mathrm{y}=1 \\
\mathrm{u}_{1}=-\bar{\varepsilon}^{i \theta} \mathrm{u}_{0}^{\prime}, \mathrm{v}=0 \text { on } \mathrm{y}=-1
\end{array}\right\}
$$

where a prime denotes differentiation with respect to $y$ and $\alpha=\bar{\varepsilon} \varepsilon(\bar{\varepsilon} \neq 0(1 / \varepsilon))$. Introducing the stream function $\bar{\psi}$ defined by

$$
\mathrm{u}_{1}=-\bar{\psi}_{\mathrm{y}}, \quad \mathrm{v}_{1}=\bar{\psi}_{\mathrm{x}}
$$

into (2.11) and (2.12) and eliminating the dimensionless pressure, we obtain

$$
\mathrm{Ru}_{0}\left(\bar{\psi}_{\mathrm{xxx}}+\bar{\psi}_{\mathrm{xyy}}\right)-\mathrm{R} \mathrm{u}_{0}^{\prime \prime} \bar{\psi}_{\mathrm{x}}=\bar{\psi}_{\mathrm{xxxx}}+2 \bar{\psi}_{\mathrm{xxyy}}+\bar{\psi}_{\mathrm{yyyy}} \cdot
$$

Assuming $\bar{\psi}(\mathrm{x}, \mathrm{y})=\varepsilon \mathrm{e}^{\mathrm{ikx}} \psi(\mathrm{y})$,

from which we infer

$$
\begin{aligned}
& u_{1}(x, y)=\varepsilon e^{i k x} \bar{u}_{1}(y), \\
& v_{1}(x, y)=\varepsilon e^{i k x} \bar{v}_{1}(y),
\end{aligned}
$$

and using (2.18) in (2.17), we obtain

$$
\psi^{i v}+i k R\left(u_{0}^{\prime} \psi-u_{0} \psi^{\prime \prime}\right)-2 k^{2} \psi^{\prime \prime}+i k^{3} \mathrm{Ru}_{0} \psi+k^{4} \psi=0 .
$$

The boundary conditions (2.15) can be now written in terms of $\psi$ as

$$
\left.\begin{array}{l}
\psi^{\prime}=\mathrm{u}_{0}^{\prime}, \quad \psi=0 \text { on } \mathrm{y}=1, \\
\psi^{\prime}=\bar{\varepsilon}^{i \theta} \mathrm{u}_{0}^{\prime}, \quad \psi=0 \text { on } \mathrm{y}=-1 .
\end{array}\right\}
$$

Equation (2.19) subject to the boundary conditions $(2.20)$ has been integrated numerically by the method of superposition coupled with an orthonormalization procedure and a variable-step Runge-Kutta-Fehlberg integration scheme. Also, an analytical solution has been obtained, within the long-wave approximation, and these results are compared with the numerical ones, for the skin friction and pressure drop (see Figure 1).

\section{ZEROTH-ORDER SOLUTION (MEAN PART).}

The solution for the zeroth-order velocity $u_{0}$, satisfying the differential equation (2.10) and the boundary conditions (2.14), has been obtained but is not presented here. The expression for $u_{0}$ at various values of $y$ has been evaluated numerically for several sets of values of the parameters $R$ and $C$. These results were found to be in good agreement with those of the generalized Couette flow. 


\section{SKIN FRICTION.}

The shear stress $\tilde{\tau}_{x y}$ at any point in the fluid is given by

$$
\tilde{\tau}_{\mathrm{xy}}=\mu *\left(\partial u *\left|\partial \mathrm{y}^{*}+\partial \mathrm{v} *\right| \partial \mathrm{x}^{*}\right) \text {. }
$$

In dimensionless form this becomes

$$
\tau_{x y}=\frac{\tilde{\tau}_{x y} d \star^{2}}{\rho^{\star} v^{\star 2}}=(\partial u|\partial y+\partial v| \partial x) .
$$

At the wavy walls $y=Y_{1}$ and $y=Y_{2}, \tau_{x y}$ becomes

$$
\begin{aligned}
& \tau_{1}=\tau_{1}^{0}+\varepsilon e^{i k x}\left[u_{0}^{\prime \prime}(1)-\psi^{\prime \prime}(1)\right], \\
& \tau_{2}=\tau_{2}^{0}+\varepsilon e^{i k x}\left[\bar{\varepsilon} e^{i \theta} u^{\prime \prime}(-1)-\psi^{\prime \prime}(-1)\right]
\end{aligned}
$$

respectively, where

$$
\tau_{1}^{0}=u_{0}^{\prime}(1) \text { and } \tau_{2}^{0}=u_{0}^{\prime}(-1)
$$

To study the effects of wall waviness on friction, in general, we can rewrite the expressions $(4.1)$ and $(4.2)$ as

$$
\begin{aligned}
& \tau_{1}=\tau_{1}^{0}+\varepsilon e^{i k x}\left|F_{1}\right| e^{i \phi_{1}}, \\
& \tau_{2}=\tau_{2}^{0}+\varepsilon e^{i k x}\left|F_{2}\right| e^{i \phi_{2}}
\end{aligned}
$$

and, in particular, hope to bring out the salient features of $\left|F_{1}\right|,\left|F_{2}\right|, \phi_{1}$, and $\phi_{2}$ in this paper (see Figures $2,3,5,6,8$ and 9).

The expressions for $\tau_{1}^{0}$ and $\tau_{2}^{0}$ have been obtained from the zeroth-order solution and have been evaluated numerically for several sets of values of the parameters $R$ and $C$. It is clear that expression (4.3) is the zeroth-order skin friction at the walls and that its numerical values correspond physically to the behavior of the flow at the walls in the case of a channel whose walls are flat (generalized Couette flow).

5. PRESSURE-DROP.

We refer to (2.5) and (2.6) and obtain the fluid pressure 


$$
\begin{array}{r}
p(x, y)=\int d p=\int\left[\frac{\partial p}{\partial x} d x+\frac{\partial p}{\partial y} d y\right], \\
\text { or } \quad p(x, y)-L=i \varepsilon\left[\frac{\exp (i k x)}{k} z(y)\right]
\end{array}
$$

where $L$ is an arbitrary constant and

$$
\mathrm{Z}(\mathrm{y})=\frac{1}{\mathrm{R}}\left(\psi^{\prime \prime},-\mathrm{k}^{2} \psi^{\prime}\right)-i \mathrm{k}\left(\mathrm{u}_{0} \psi^{\prime}-\mathrm{u}_{0}^{\prime} \psi\right) \text {. }
$$

Equation (5.1) can be written as

$$
\begin{aligned}
\hat{\mathrm{p}} & =\mathrm{p}(\mathrm{x},-1)-\mathrm{p}(\mathrm{x}, 1)=\frac{i \varepsilon \mathrm{e}^{i k x}}{\mathrm{k}}[\mathrm{z}(-1)-z(1)] \\
\text { or } \hat{\mathrm{p}} & =\varepsilon \mathrm{e}^{i k \mathrm{x}}|\mathrm{Q}| \mathrm{e}^{i \beta},
\end{aligned}
$$

where $\hat{p}$ has been referred to as the pressure drop since it indicates the difference in the pressure on the wavy walls, with $x$ fixed. The amplitude $|Q|$ and the phase $\beta$ were evaluated numerically for several sets of values of the dimensionless parameters entering the problem and are presented in Figures 4, 7, and 10. In what follows, we record the qualitative differences in the behavior of the flow characteristics that show clearly the effects of the wavy walls of the channels.

\section{RESULTS AND DISCUSSION.}

As mentioned in the introduction, the analytical solution of $\left|F_{i}\right|$, $\phi_{i}(i=1,2),|Q|$, and $\beta$ are compared with the numerical ones in Figure 1. From Figure 1 it is evident that the analytical and numerical solutions are in good agreement, for small and moderate Reynolds numbers. However, at high Reynolds numbers, the analytical solution differs very much from the numerical solution. In Figures 2-10 only the numerical solution results for the amplitudes and phases (arising from wall waviness) are plotted for various values of $R, C$, and $k$.

Figure 2 shows the behavior of $\left|F_{1}\right|$ and $\phi_{1}$ at various Reynolds numbers in the four different channels considered. Similar results for $\left(\left|\mathrm{F}_{2}\right|, \phi_{2}\right)$ and $(|Q|, B)$ are presented in Figures 3 and 4 , respectively. From Figure 2 , it follows that the amplitude $\left|F_{1}\right|$ increases with $R$ : physically it means that 
the amplitude increases when the wall moves faster. But the phase $\phi_{1}$ increases with $\mathrm{R}$ when $\theta$ is $\pi / 2$ and decreases when $\theta$ is $3 \pi / 2$. On a keen perusal into Figures 2, 3, and 4, we arrive at the conclusion that the features of $\left(\left|F_{2}\right|,|Q|\right)$ and $\left(\phi_{2}, \beta\right)$ resemble those of $\left|F_{1}\right|$ and $\phi_{1}$, respectively, in all the channels considered; the only exception is that $\beta$ decreases with $R$ for all values of $\theta$. However, the amplitudes $\left|F_{1}\right|,\left|F_{2}\right|,|Q|$ and the phases $\phi_{1}$, $\phi_{2}, \beta$ are quantitatively different.

Figure 5 describes the behavior of the amplitude $\left|F_{1}\right|$ and the phase $\phi_{1}$ for different values of C (i.e., for flows with adverse and favorable pressure gradients). From this figure we observe that $\left|F_{l}\right|$ is minimum when $C$ is 0 but $\left|F_{1}\right|$ increases with an increase in $|C|$. However, $\phi_{1}$ is minimum when $\theta$ is $\pi / 2$ and is maximum when $C$ is 0 . On comparing figures 5,6 and 7 , we note that the features of $\left(\left|F_{2}\right|,|Q|\right)$ and $\left(\phi_{2}, \beta\right)$ resemble qualitatively those of $\left|F_{2}\right|$ and $\phi_{1}$, with the exception that $\beta$ is minimum when $\theta$ is 0 and maximum when $\theta$ is $\pi$.

Figure 8 depicts the behavior of $\left|F_{1}\right|$ and $\phi_{1}$ with changes in the wavenumber. From this firgure, it is clear that the effect of wavenumber on the amplitude $\left|F_{1}\right|$ is to increase it considerably and this is true in all the channels considered, but the phase $\phi_{1}$ increases when $\theta$ is $\pi / 2$ and decreases when $\theta$ is $3 \pi / 2$. The observation from Figures 9 and 10 shows that the effect of wavenumber is to increase the amplitudes $\left|F_{2}\right|$ and $|Q|$. However, the phase $\beta$ increases (with an increase in k) in all the channels and $\phi_{2}$ increases when $\theta$ is $3 \pi / 2$ and decreases when $\theta$ is $\pi / 2$. From all these figures, it is clear that the amplitudes and the phases are very much affected (in all the channels) by the parameters $R, C$, and $k$, an indication that the skin friction (at both walls) and the pressure drop are significant1y affected not only by the usual parameters $R$ and $C$ but also by the parameters $k$ and $\theta$. Hence, it is evident that the wall waviness does affect the skin friction and the pressure drops significantly. 

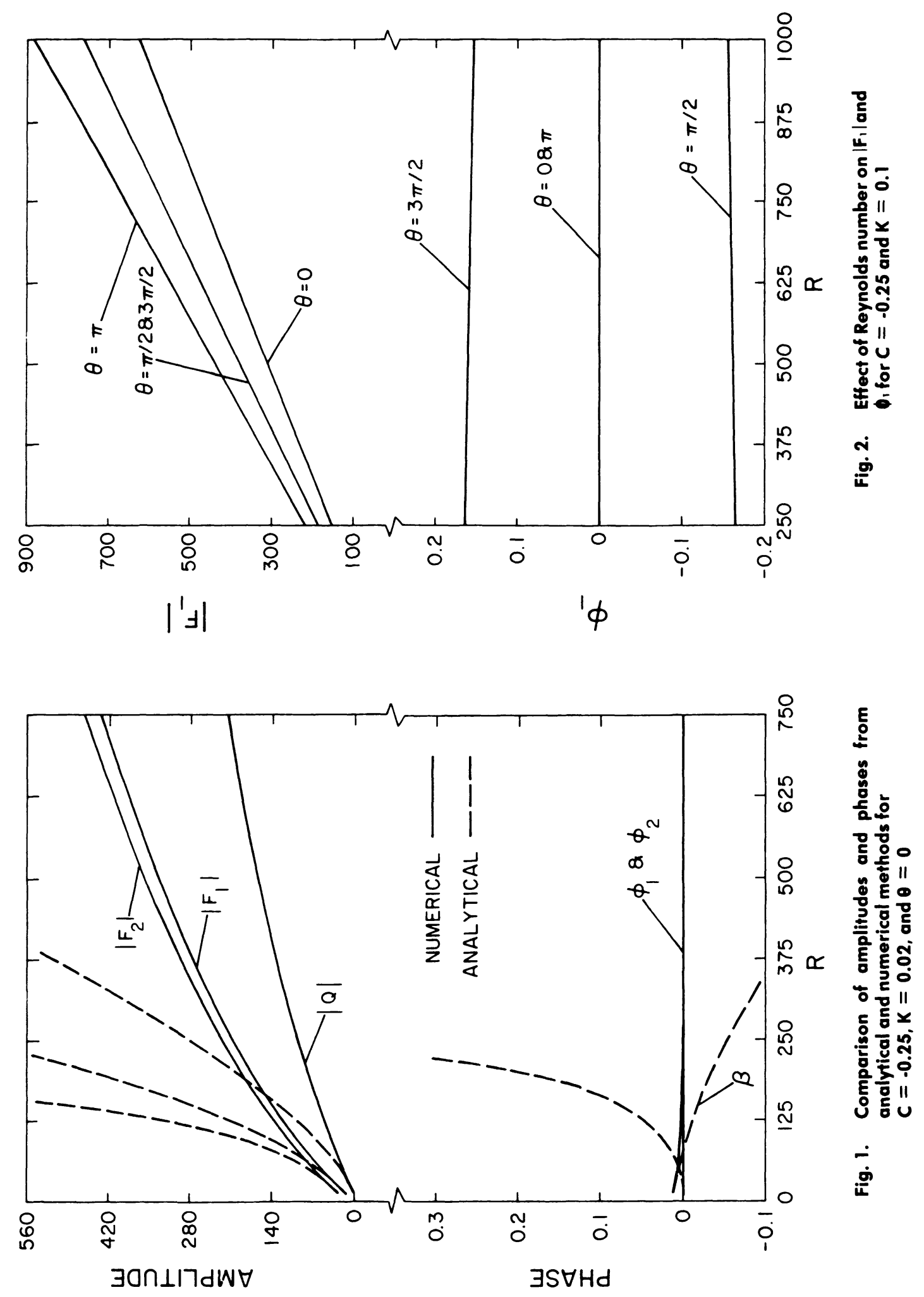

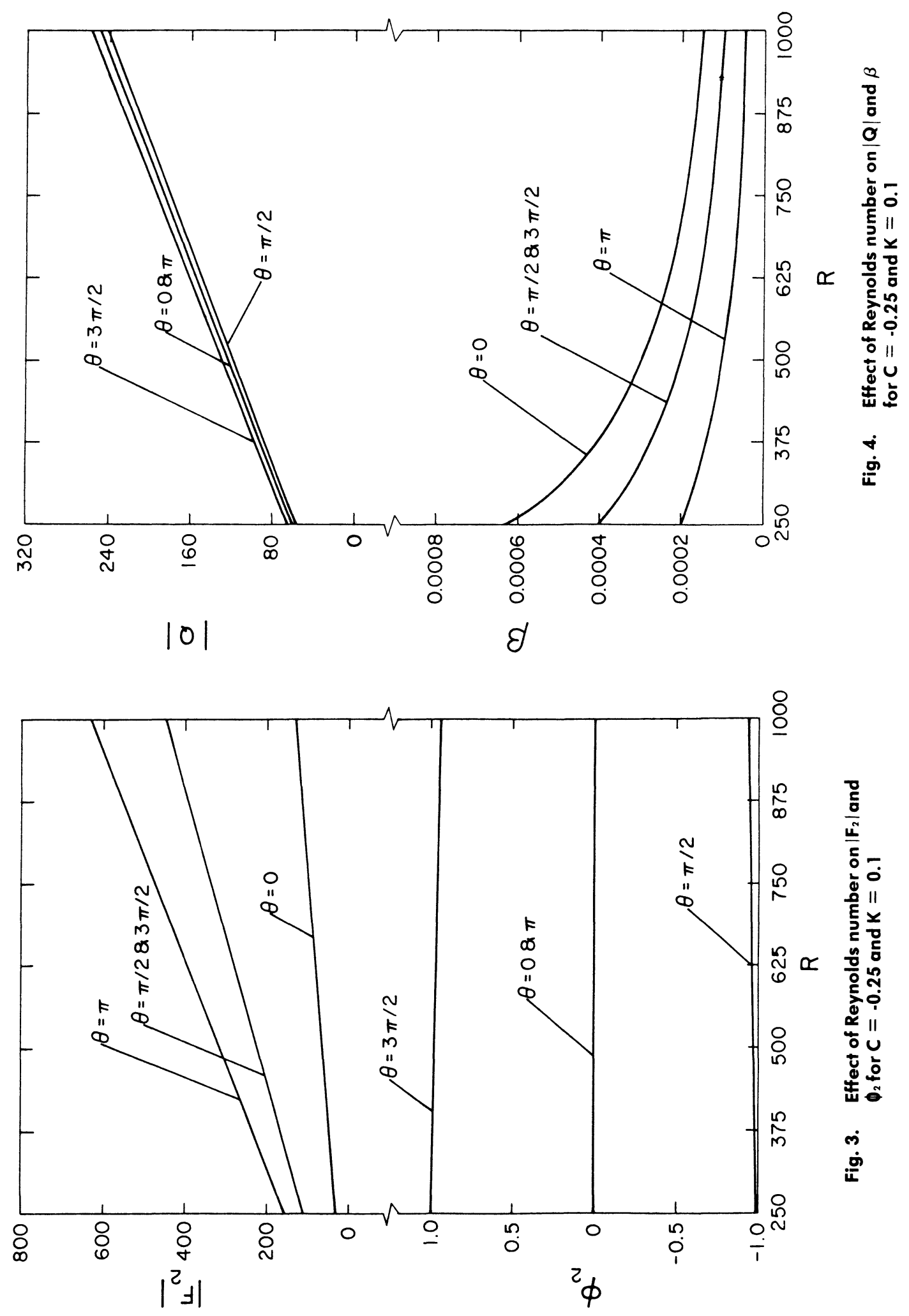

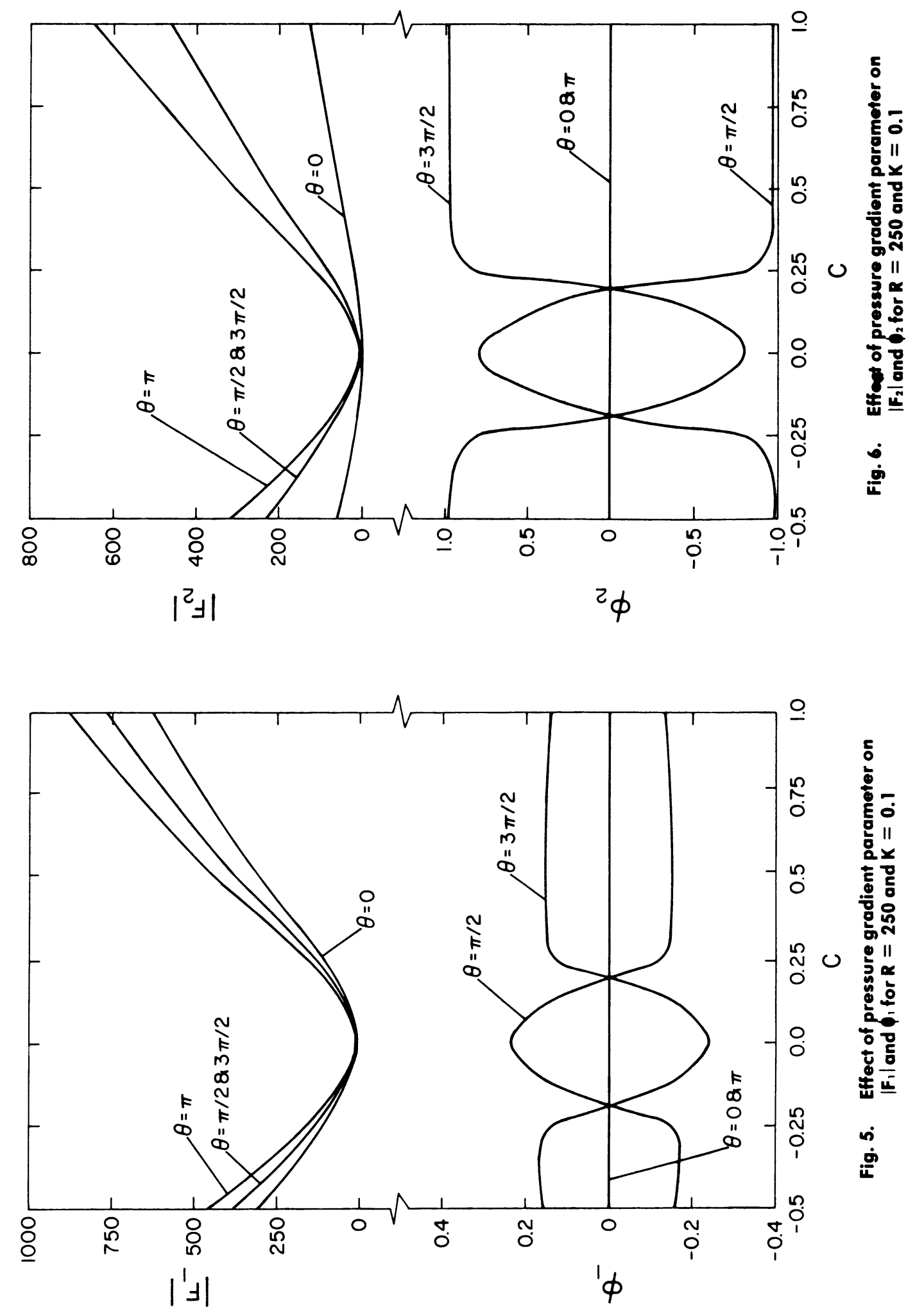

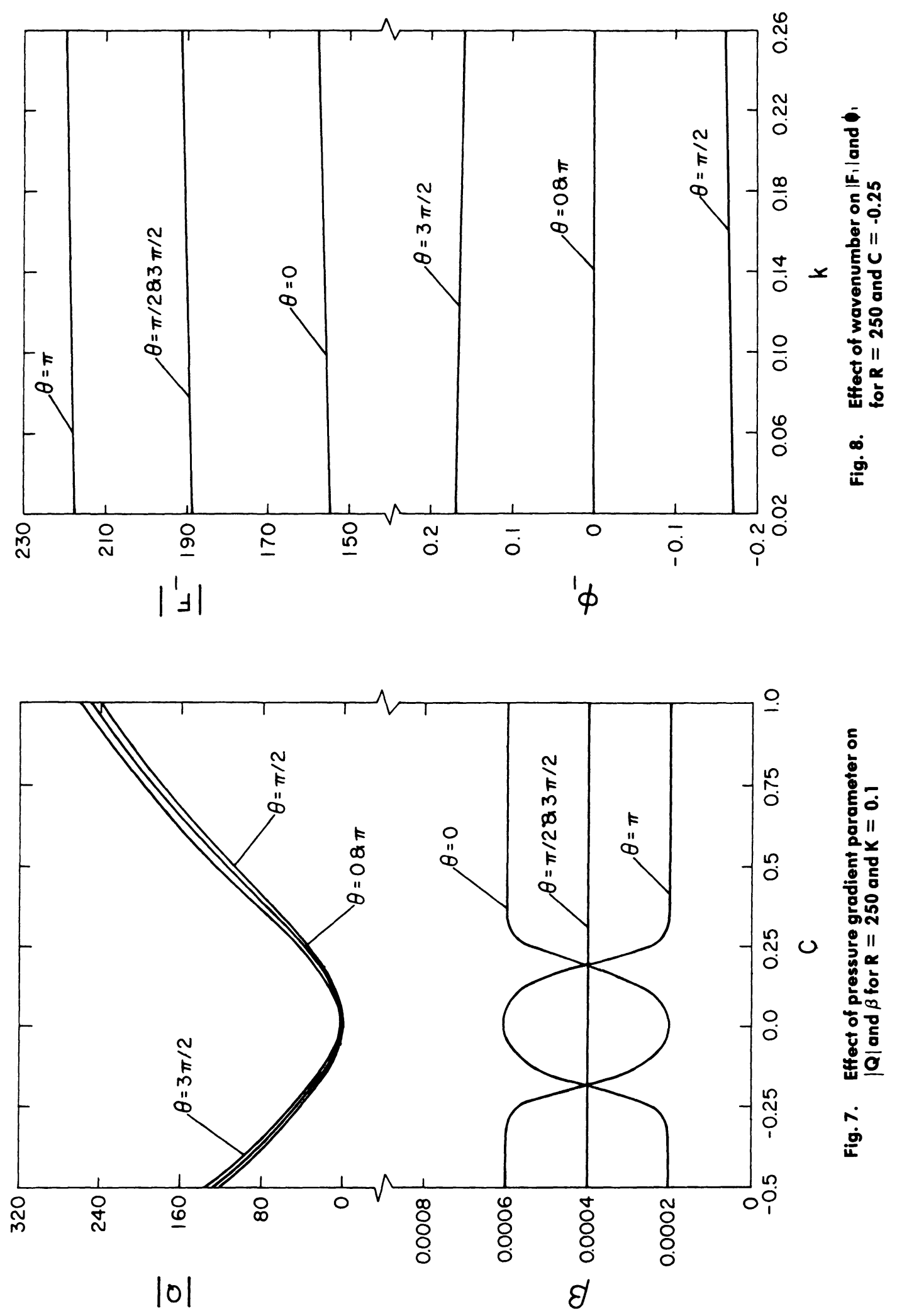

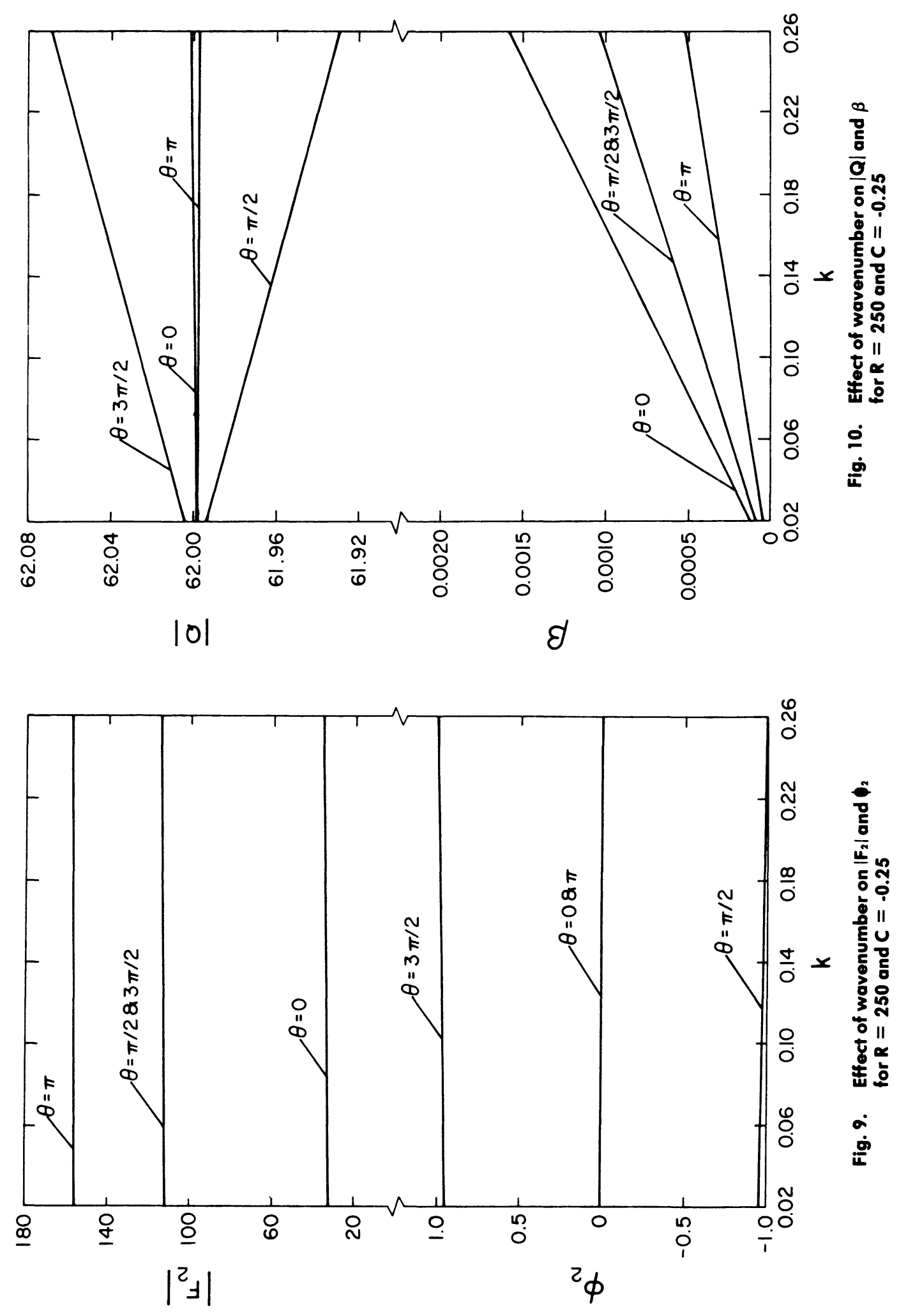


\section{CONCLUSION.}

The amplitudes $\left|F_{1}\right|,\left|F_{2}\right|$, and $|Q|$ are enhanced significantly with an increase in either the Reynolds number $R$ or the pressure gradient parameter $C$ or the wavenumber $\mathrm{k}$, in almost all the channels considered. Of all the parameters, the Reynolds number and the pressure-gradient parameter have the strongest influences on the amplitudes. However, the phases $\phi_{1}, \phi_{2}$, and $\beta$ were found to have different trends in different channels, depending on the parameters $R$, $C$ and $k$; they are very much affected by the parameters $R$ and $C$. Finally, it may be concluded that the present analysis is capable of providing us with information relating to the effects of wall-waviness on friction and pressure drop for long as well as short waves at small, moderate, and large Reynolds numbers.

\section{REFERENCES}

1. BERMAN, A. S., Laminar flows in an annulus with porous walls, J. Appl. Phys. $\underline{29}(1958), 71-75$.

2. BALARAM, M., Heat generated by MHD Couette flow with porous walls, Lubrication Tech., Trans. ASME, Series F, 97 (1975) 630-634.

3. HAHN, E. J., \& KETTLEBOROUGH, C. E., Solution for pressure and temperature in an infinite slider bearing of arbitrary profile, Lubrication Tech., Trans. ASME, Series F, 89 (1967) 445-452.

4. SZERI, A. Z., YATES, C. C., \& HAI, S. M., Flow development in a parallel plate channel, Lubrication Tech., Trans. ASME, Series F, 98 (1976) $145-155$.

5. KRAGELSKII, I. V., Friction and Wear, Butterworth, London, 1965.

6. SCOTT, M. R., \& WATTS, H. A., Support - A computer code for two point boundary-Value Problems via Orthonormalization, SAND 75-0198, 1975.

7. NAYFEH, A. H., Perturbation Methods, John Wiley \& Sons, New York, 1973. 


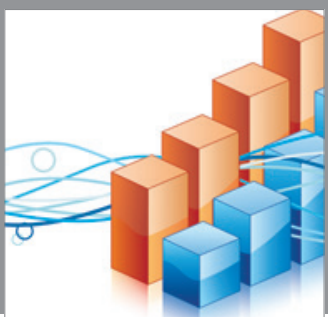

Advances in

Operations Research

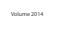

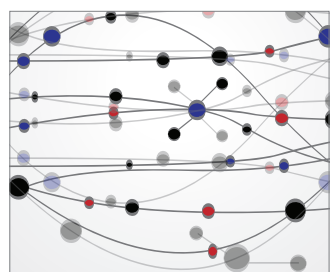

\section{The Scientific} World Journal
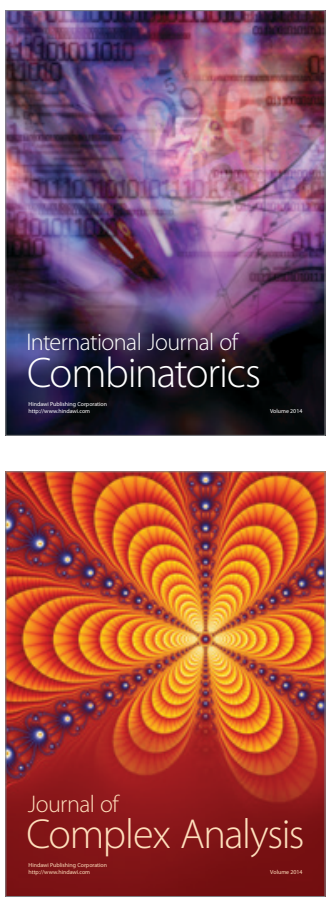

International Journal of

Mathematics and

Mathematical

Sciences
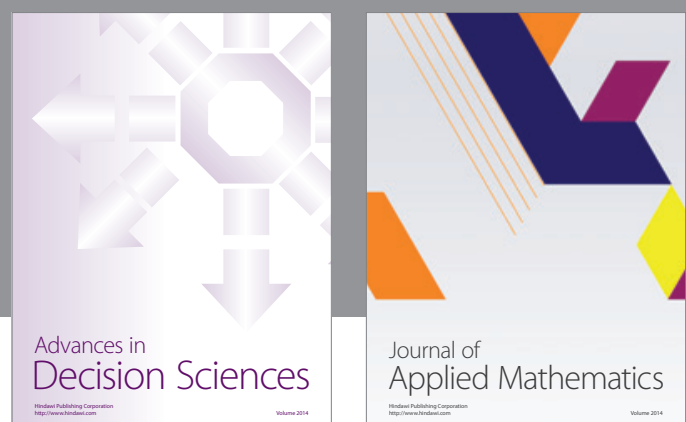

Journal of

Applied Mathematics
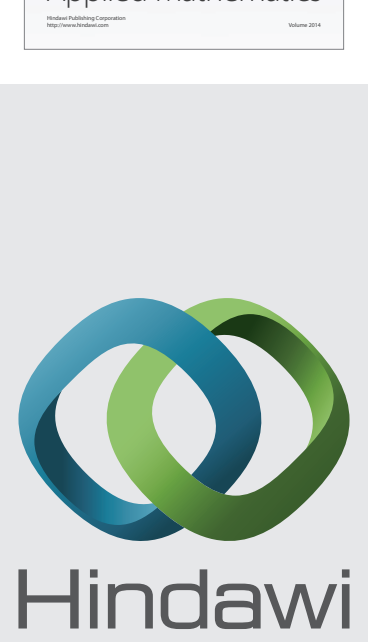

Submit your manuscripts at http://www.hindawi.com
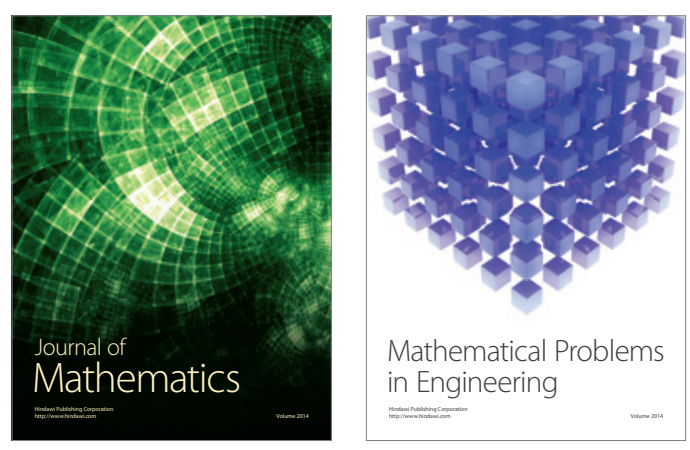

Mathematical Problems in Engineering
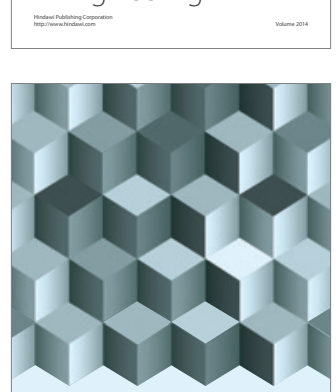

Journal of

Function Spaces
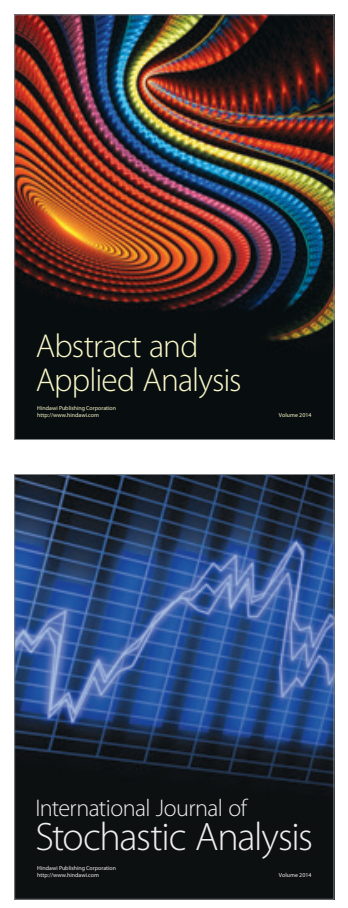

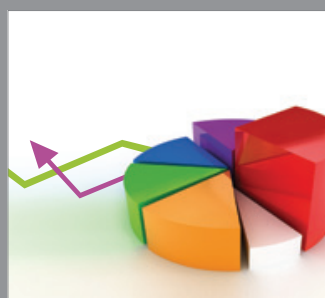

ournal of

Probability and Statistics

Promensencen
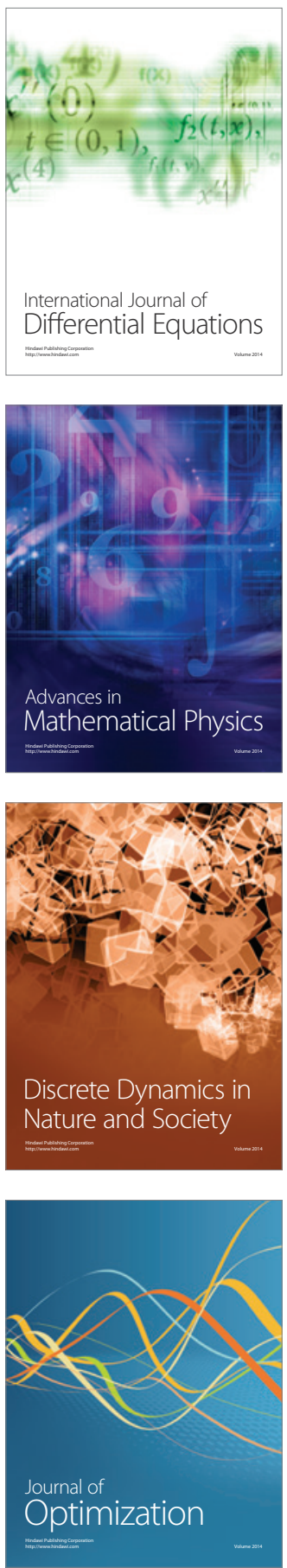